\title{
Role of glucagon-like peptide-2 deficiency in neonatal short-bowel syndrome using neonatal piglets
}

\author{
Zheng Hua', Justine M. Turner 1,2, David L. Sigalet ${ }^{3}$, Pamela R. Wizzard', Patrick N. Nation', Diana R. Mager ${ }^{1,2}$, Ron O. Ball'2, \\ Paul B. Pencharz ${ }^{5-7}$ and Paul W. Wales ${ }^{1,5,8,9}$
}

BACKGROUND: Short-bowel syndrome (SBS) is the most common cause of neonatal intestinal failure. Recovery requires intestinal adaptation, dependent on enteral nutrition (EN) and growth factors such as glucagon-like peptide-2 (GLP-2), which is secreted from $L$ cells in the ileum. Neonatal SBS often results in loss of ileum; therefore, we hypothesized that without ileum, endogenous GLP-2 production would be inadequate to promote adaptation. We compared endogenous GLP-2 production and adaptation in neonatal animals with SBS, with and without ileum.

METHODS: Neonatal piglets (4-6 d) were randomized to 75\% mid-intestinal resection, 75\% distal-intestinal resection, or sham control without resection. Postoperatively, all piglets commenced parenteral nutrition (PN), tapering as EN was increased to maintain specific growth.

RESULTS: The resected SBS piglets developed intestinal failure, requiring a longer duration of PN support and experiencing fat malabsorption. The piglets without ileum were not able to wean from PN during the study and did not show adaptation, specifically growth in intestinal length or crypt hyperplasia on histology of the jejunum. Adaptation was observed in the resected SBS piglets with ileum, and these piglets also had an increased plasma GLP-2 level that was not observed in piglets without ileum.

CONCLUSION: SBS piglets with ileum undergo adaptation associated with increased endogenous GLP-2 production. SBS piglets without ileum undergo limited adaptation and severe intestinal failure, requiring prolonged PN support. This appears to be related to a deficiency in endogenous GLP-2 production.

$\mathbf{N}$ eonatal intestinal failure secondary to short-bowel syndrome (SBS) has a particularly poor outcome. It is most common in preterm infants, and necrotizing enterocolitis is the most common cause (1-3). Necrotizing enterocolitis usually results in loss of ileum and right colon and carries a significant mortality (4).

After intestinal resection, adaptation of the remnant intestine is essential for survival, compensating for the reduced absorptive surface area, both structurally and functionally (5). This process is regulated by several factors, including the stage of intestinal development, site and length of resection, the underlying disease, nutritional status, and gut-derived hormones and growth factors (6). Among the growth factors, glucagon-like peptide-2 (GLP-2) is considered to have an important role in inducing small-intestinal adaptation after resection. GLP-2 acts specifically at the GLP-2 receptor (GLP-2R), which is expressed throughout the small and large intestines but with greatest concentration in the jejunum (7). GLP-2 is uniquely trophic for the intestine (8). It can stimulate residual small-intestinal adaptation by increasing crypt cell proliferation and inhibiting apoptosis, thereby increasing villus height, crypt depth, mucosal mass, and overall small-intestinal length and weight (9-11). Functionally, GLP-2 increases activities of mucosal enzymes and therefore increases digestion and absorption (12).

Endogenous GLP-2 is secreted from the neuroendocrine L-cells in the subepithelium, with highest concentration in the distal ileum and proximal colon (13). Endogenous release is induced by both proximal enteric neuronal signaling and direct stimulation from nutrients in the distal bowel, especially long-chain fatty acids (14-17). Because necrotizing enterocolitis and other common causes of neonatal SBS are often associated with damage to or loss of ileum, we hypothesized that deficient endogenous GLP-2 release is a factor contributing to poor outcomes in neonates with SBS, by limiting adaptation $(13,18)$.

We previously developed neonatal piglet SBS models with and without ileal resection (19). Neonatal piglets have gastrointestinal anatomy, physiology, and metabolism similar to those of human neonates $(20,21)$. Piglets have less intrauterine gut development and hence at birth are more similar to premature neonates (20). Because premature babies are 14 times more likely to experience SBS, this is a clinically relevant translational model (20).

We compared plasma GLP-2 levels in the piglet models with equivalent resection length but varied remnant anatomy. 
We studied functional adaptation, including duration of parenteral nutrition (PN) support to maintain growth and fecal fat absorption, and structural intestinal adaptation, including intestinal lengthening and histology.

\section{RESULTS}

\section{Piglet Performance}

Short-bowel piglets had the equivalent of a $75 \%$ small-intestine resection, whereas the sham piglets did not have resection. The jejunoileal (JI) had remaining ileum, whereas the jejunocolic (JC) did not have ileum remaining. Overall, piglets in the JI and sham groups were active and healthy, whereas piglets in the JC group were often lethargic. On the basis of fever and/or persistent vomiting, catheter sepsis was suspected and blood cultures were taken. Positive blood culture results were found in two JI piglets $(2 / 16,12.5 \%)$, five JC piglets $(5 / 17,29.4 \%)$, and two sham piglets $(2 / 15,13.3 \%)$.

Weight gain was greater in sham piglets as compared with JC piglets (Figure 1a). The duration of PN support was longer in the SBS piglets than sham piglets, and longest in the JC group $(P<0.05$; Table 1$)$. All 15 sham piglets $(100 \%)$ and 11 of $16 \mathrm{JI}$ piglets (68.8\%) were able to discontinue PN, but only 1 of 17 JC piglets (or 5.9\%) was able to stop PN support. JC piglets had the least ability to tolerate enteral nutrition (EN), as compared with the JI and sham piglets $(P<0.05$; Table 1$)$. The EN tolerated is shown in Figure $\mathbf{1 b}$.

\section{Fat Absorption}

Table 1 shows enteral lipid delivery and fat absorption in each surgical group during both fecal collections. During both collections, enteral fat infusion in JI and sham piglets was the same and was higher than that of JC piglets. The absolute amount of enteral fat absorbed in grams was different between groups: highest in the sham and lowest in the JC piglets $(P<$ $0.05)$. When expressed as fat absorbed per final length of small bowel, $\mathrm{g} / \mathrm{kg} / \mathrm{d} / \mathrm{cm}$, during both collections, JI piglets had the highest and sham piglets had the lowest absorption per $\mathrm{cm}$.

\section{Plasma GLP-2 Levels}

GLP-2 levels increased from the baseline to the end of trial in JI piglets (Figure 2). At the end of trial, no difference was found in plasma GLP-2 levels between JC and sham piglets. In JC piglets, plasma GLP-2 levels increased only from baseline to the second collection, while receiving $50 \%$ total energy enterally, whereas no increase occurred at the end of the trial. Plasma GLP-2 level did not change throughout the trial in shams. When plasma GLP-2 level is expressed per length of final small intestine, JI piglets had the highest, JC piglets intermediate, and sham piglets the lowest (JI: $0.38 \pm 0.05 \mathrm{pmol} / \mathrm{l}$, JC: $0.20 \pm 0.04 \mathrm{pmol} / \mathrm{l}$, sham: $0.04 \pm 0.01 \mathrm{pmol} / \mathrm{l}, P<0.05)$ values. By linear regression, the amount of EN correlated with the final circulating GLP-2 level $\left(R^{2}=0.407, P<0.05\right)$. In addition, the duration of $\mathrm{PN}$ support also correlated with the circulating GLP-2 level $\left(R^{2}\right.$ linear $\left.=0.312, P<0.05\right)$.

\section{Small-Intestinal Growth and Structural Adaptation}

Absolute small-intestinal length over the trial increased more in sham piglets, as compared with JI piglets, and did not increase in JC piglets $(P<0.05$; Table 2$)$. Expressed as a percentage of postresection length, JI piglets and sham piglets demonstrated similar intestinal lengthening $(P<0.05$; Table 2$)$. Similarly, dry small-bowel weight was highest in sham piglets and lowest in JC piglets $(P<0.05$; Table 2$)$. The weight of the small intestine per unit length was greater in JI piglets than in JC and sham

Table 1. Clinical observation of piglets

\begin{tabular}{|c|c|c|c|c|}
\hline & $\mathrm{Jl}(n=16)$ & $\mathrm{JC}^{\mathrm{b}}(n=17)$ & $\operatorname{Sham}^{c}(n=15)$ & $P$ \\
\hline \multicolumn{5}{|l|}{ EN tolerance } \\
\hline Days of PN support & $10.3 \pm 0.7^{\mathrm{b}, \mathrm{c}}$ & $13.4 \pm 0.4^{\mathrm{a}, \mathrm{c}}$ & $6.1 \pm 0.3^{a, b}$ & $<0.05$ \\
\hline Highest EN tolerated (\% total energy) & $88.1 \pm 4.6^{\mathrm{b}, \mathrm{c}}$ & $60.0 \pm 3.0^{\mathrm{a}, \mathrm{c}}$ & $100^{\mathrm{a}, \mathrm{b}}$ & $<0.05$ \\
\hline \multicolumn{5}{|l|}{ Fat absorption } \\
\hline Enteral lipid delivered (g/kg/d) & $5.3 \pm 0.4^{b}$ & $3.9 \pm 0.3^{\mathrm{a}, \mathrm{c}}$ & $6.0 \pm 0.3^{b}$ & $<0.05$ \\
\hline Fat absorbed $(\mathrm{g} / \mathrm{kg} / \mathrm{d})$ & $4.6 \pm 0.3^{b, c}$ & $2.9 \pm 0.3^{a, c}$ & $5.7 \pm 0.4^{a, b}$ & $<0.05$ \\
\hline Fat absorption (\% of total lipid delivered) & $85.6 \pm 2.1^{\mathrm{b}, \mathrm{c}}$ & $73.3 \pm 3.4^{\mathrm{a}, \mathrm{c}}$ & $94.8 \pm 2.7^{\mathrm{a}, \mathrm{b}}$ & $<0.05$ \\
\hline Fat absorption $(\mathrm{g} / \mathrm{kg} / \mathrm{d} / \mathrm{cm})$ & $0.029 \pm 0.002^{\mathrm{b}, \mathrm{c}}$ & $0.019 \pm 0.002^{\mathrm{a}, \mathrm{c}}$ & $0.009 \pm 0.001^{\mathrm{a}, \mathrm{b}}$ & $<0.05$ \\
\hline \multicolumn{5}{|l|}{ Day 12} \\
\hline Fat absorption $(\mathrm{g} / \mathrm{kg} / \mathrm{d} / \mathrm{cm})$ & $0.025 \pm 0.002^{\mathrm{b}, \mathrm{c}}$ & $0.017 \pm 0.002^{\mathrm{a}, \mathrm{c}}$ & $0.010 \pm 0.001^{\mathrm{a}, \mathrm{b}}$ & $<0.05$ \\
\hline
\end{tabular}

Values were expressed as mean \pm SE. P values refer to comparison among the three groups (Jl, JC, and sham) using ANOVA.

EN, enteral nutrition; JC, jejunocolic; Jl, jejunoileal; PN, parenteral nutrition.

a,b,cBetween-group comparisons using independent samples Student's $t$-test, $P<0.05$. 


\section{Articles | Hua et al.}
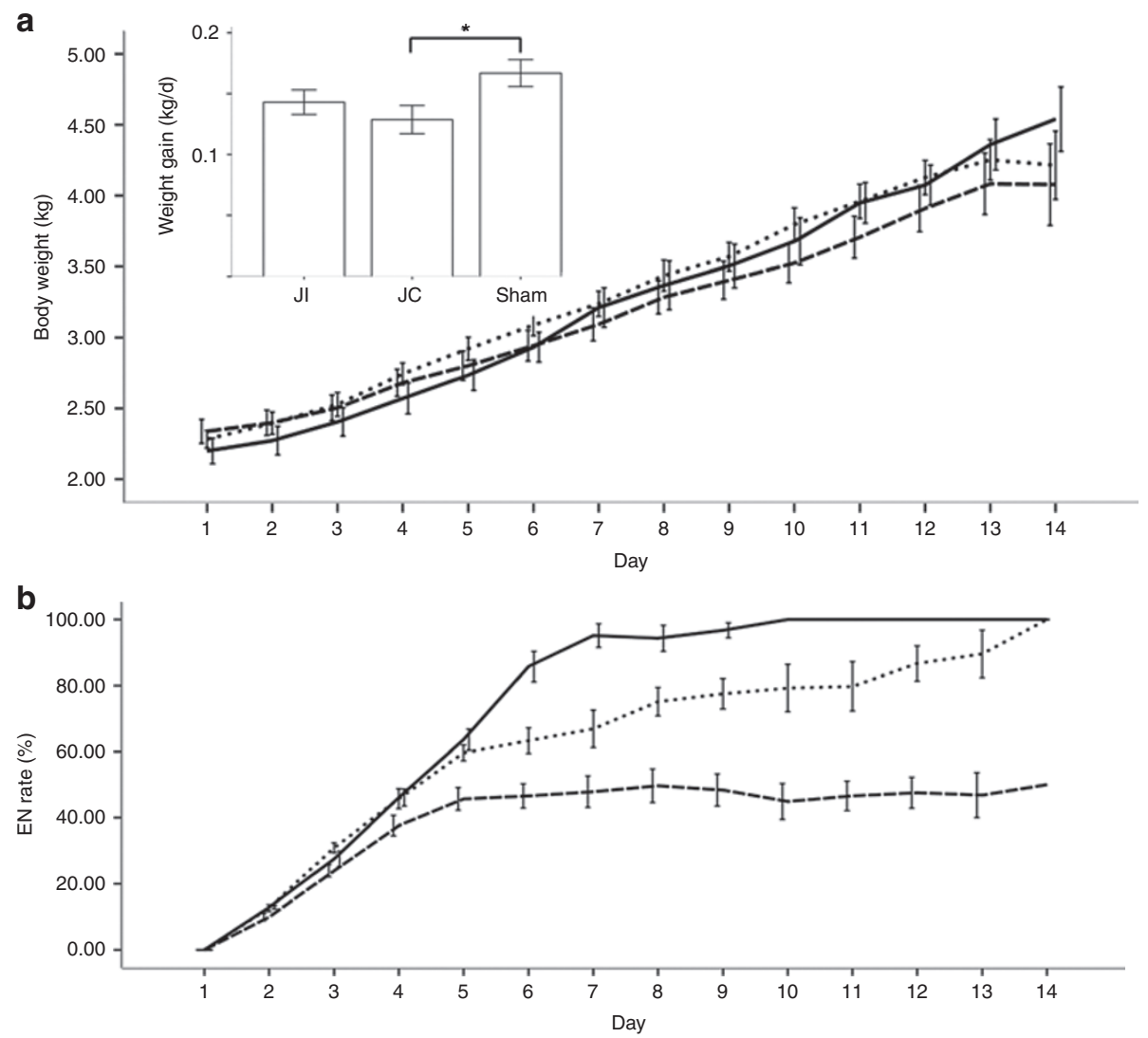

Figure 1. Weight gain and enteral nutrition intake of piglets, according to group. (a) Daily weight gain of piglets ( $\mathrm{kg} / \mathrm{d}$ ) on trial (mean $\pm \mathrm{SE}$ ). Jl piglets represented by the dotted line, $n=16$; JC piglets represented by the dashed line, $n=17$; sham piglets represented by the solid line, $n=15$. Sham piglets gained more weight than short-bowel syndrome piglets. Comparison between two surgical groups done using independent samples Student's $t$-test, ${ }^{*} P$ $<0.05$. (b) Daily EN rate (as percentage of total energy, \%) on trial (mean \pm SE). Jl piglets represented by the dotted line, $n=16$; JC piglets represented by the dashed line, $n=17$; sham piglets represented by the solid line, $n=15$. Sham piglets received $100 \%$ of nutrients enterally at the end of trial, whereas most JC piglets could tolerate only a maximum of $50 \%$ nutrient delivery by the enteral route. EN, enteral nutrition; JC, jejunocolic; J, jejunoileal.

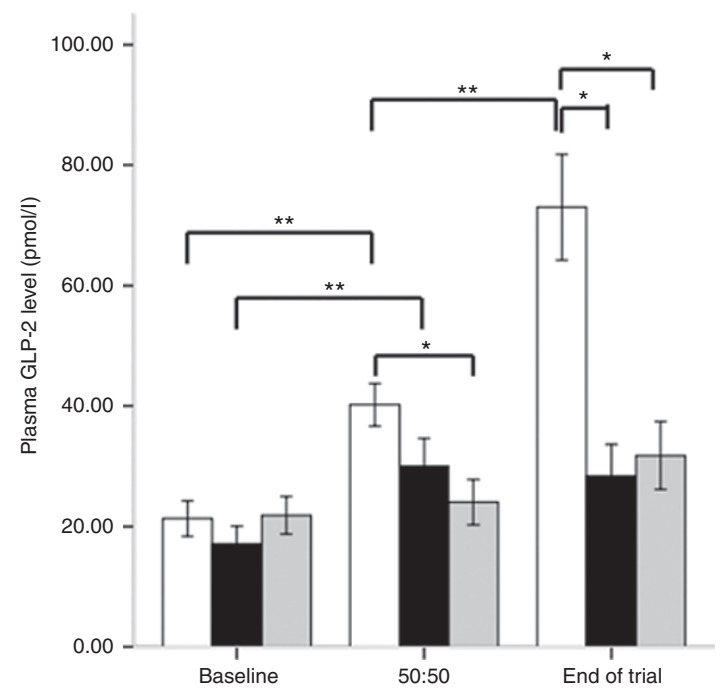

Figure 2. Plasma GLP-2 level ( $\mathrm{pmol} / \mathrm{l}$; mean $\pm \mathrm{SE}$ ) at the three time points: baseline, when on $50 \%$ enteral nutrition (50:50), and at the end of the trial. $\mathrm{Jl}$ piglets represented by white bar, $n=16$; JC piglets represented by black bar, $n=17$; sham piglets represented by gray bar, $n=15$. Comparison between two surgical groups done using independent samples Student's $t$-test, ${ }^{*} P<0.05$; within-group comparisons, at different time points, done using paired-samples $t$-test, ${ }^{* *} P<0.05$. GLP-2, glucagon-like peptide-2; $J C$, jejunocolic; J, jejunoileal. piglets (JI: $0.46 \pm 0.04 \mathrm{~g} / \mathrm{cm}$, JC and sham: $0.25 \pm 0.02 \mathrm{~g} / \mathrm{cm}, P$ $<0.05)$.

Histological evidence of adaptation was notable in JI piglets, with higher villi and deeper crypts in the ileum, and jejunal crypt hyperplasia $(P<0.05$; Table 2 , Figure 3$)$. Histological adaptation of the jejunum was not observed in JC piglets. There was no difference in apoptosis measured at either the villus or crypt among the three groups (data not shown).

\section{GLP-1, L-Cell, and GLP-2R Expression}

There were no differences between groups for L-cell staining and for GLP-1-expressing cells (known to be coexpressed with GLP-2) at any anatomical site for any group (Figures 4 and 5).

GLP-2R expression in each intestinal segment (jejunum, ileum, and colon) at the end of the trial showed no differences among the three surgical groups.

\section{Plasma GLP-2 and Structural Adaptation}

Correlations between plasma GLP-2 level at the end of trial and histologic measurement of intestinal adaptation were calculated in SBS piglets. Plasma GLP-2 levels were significantly correlated with small-intestinal lengthening through the trial (Figure 6a) and with the small-bowel dry weight (Figure 6b). 
Table 2. Structural intestinal adaptation

\begin{tabular}{|c|c|c|c|c|}
\hline & $\mathrm{Jl}^{\mathrm{a}}(n=16)$ & $\mathrm{JC}^{\mathrm{b}}(n=17)$ & $\operatorname{Sham}^{c}(n=15)$ & $P$ \\
\hline \multicolumn{5}{|l|}{ Morphological changes } \\
\hline Small-bowel length, beginning $(\mathrm{cm})$ & $619.0 \pm 20.3$ & $611.0 \pm 21.3$ & $605.2 \pm 12.2$ & 0.889 \\
\hline Small-bowel length, termination (cm) & $192.2 \pm 6.1^{\mathrm{b}, \mathrm{c}}$ & $156.3 \pm 5.9^{\mathrm{a}, \mathrm{c}}$ & $796.8 \pm 30.4^{\mathrm{a}, \mathrm{b}}$ & $<0.05$ \\
\hline Small-bowel length change $(\mathrm{cm})$ & $33.6 \pm 6.0^{b, c}$ & $-0.8 \pm 3.8^{\mathrm{a}, \mathrm{c}}$ & $191.7 \pm 28.6^{\mathrm{a}, \mathrm{b}}$ & $<0.05$ \\
\hline Small-bowel dry weight (g) & $89.7 \pm 8.4^{\mathrm{b}, \mathrm{c}}$ & $39.5 \pm 3.2^{\mathrm{a}, \mathrm{c}}$ & $191.8 \pm 10.9^{\mathrm{a}, \mathrm{b}}$ & $<0.05$ \\
\hline Small-bowel dry weight/small-bowel length $(\mathrm{g} / \mathrm{cm})$ & $0.46 \pm 0.04^{\mathrm{b}, \mathrm{c}}$ & $0.25 \pm 0.02^{\mathrm{a}}$ & $0.25 \pm 0.02^{\mathrm{a}}$ & $<0.05$ \\
\hline \multicolumn{5}{|l|}{ Histological changes } \\
\hline Jejunal villi height $\left(\times 10^{-1} \mathrm{~mm}\right)$ & $7.2 \pm 0.5^{b}$ & $5.3 \pm 0.3^{\mathrm{a}, \mathrm{c}}$ & $7.3 \pm 0.7^{b}$ & $<0.05$ \\
\hline Jejunal crypt depth $\left(\times 10^{-1} \mathrm{~mm}\right)$ & $2.0 \pm 0.1^{\mathrm{b}, \mathrm{c}}$ & $1.7 \pm 0.1^{\mathrm{a}}$ & $1.6 \pm 0.1^{\mathrm{a}}$ & $<0.05$ \\
\hline Jejunal proliferative crypt cell number & $333.8 \pm 21.7^{\mathrm{b}, \mathrm{c}}$ & $260.3 \pm 18.9^{a}$ & $242.1 \pm 18.0^{\mathrm{a}}$ & $<0.05$ \\
\hline Total jejunal crypt cell number & $1044.1 \pm 42.9^{c}$ & $973.2 \pm 52.8^{c}$ & $794.4 \pm 37.3^{\mathrm{a}, \mathrm{b}}$ & $<0.05$ \\
\hline
\end{tabular}

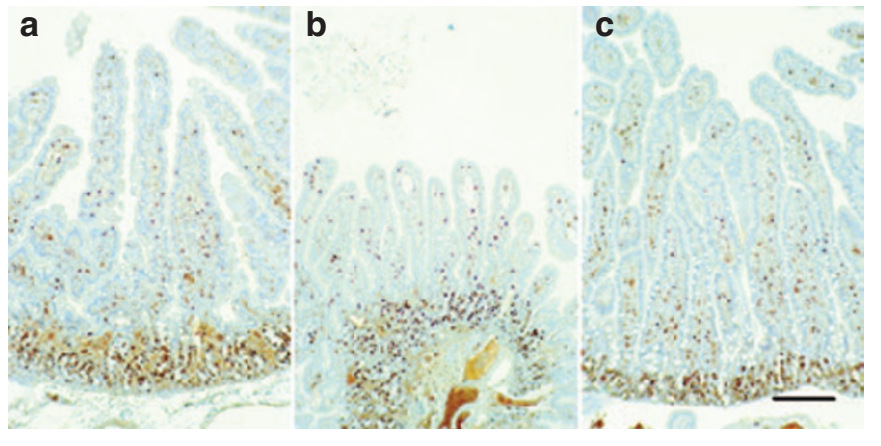

Figure 3. Photomicrographs of representative cross sections of jejunum from (a) a jejunoileal, (b) a jejunocolic, and (c) a sham piglet at $14 \mathrm{~d}$ after surgery. Original magnifications are all $\times 100$ and therefore provide a comparison of mucosal hyperplasia. This is further supported by Ki67 staining (brown). Note the prominent villa lengthening and crypt hyperplasia in a. Villus length measured is (a) $0.104 \mathrm{~mm}$, (b) $0.069 \mathrm{~mm}$, and (c) $0.087 \mathrm{~mm}$; crypt depth is (a) $0.017 \mathrm{~mm}$, (b) $0.016 \mathrm{~mm}$, and (c) $0.014 \mathrm{~mm}$. Bar $=100 \mu \mathrm{m}$.

In JI piglets, correlations between ileal histological adaptation and plasma GLP-2 levels were significant (Figure 6c).

\section{DISCUSSION}

Neonatal piglets with short bowel, but without ileum, have less endogenous GLP-2 production than SBS piglets with ileum; this is associated with lack of adaptation and normal growth of the intestine.

JI piglets, with ileum, demonstrated a progressive increase in plasma GLP-2 concentrations, associated with crypt hyperplasia, villus lengthening, and small-intestinal lengthening. In the JC piglets, without ileum, there was an increase in GLP-2 level early postresection; however, at trial end, the plasma
GLP-2 level did not elevate further and there was also neither crypt hyperplasia nor intestinal lengthening, but in contrast, there was villous atrophy. In sham piglets, without resection, plasma GLP-2 levels did not rise; crypt hyperplasia was not observed; and the intestine grew as developmentally appropriate. Expressed as a percentage increase over the remnant length at initial surgery, the changes in length for JI and sham piglets were similar. Therefore, it is reasonable to propose that JI piglets, with ileum, have normal small-intestinal growth similar to that of sham piglets, their developmentally equivalent peers. However, in JC piglets, without an ileum, developmentally appropriate growth in intestinal length did not occur.

The main sites for endogenous GLP-2 release, in both pigs and humans, are the L-cells, which are distributed predominantly in the distal ileum and colon, although present to a lesser degree in jejunum $(10,22)$. The presence of ileum is probably the reason why JI piglets, with remnant ileum, had increased plasma GLP-2 levels postresection (13). By contrast, JC piglets, with ileum and proximal colon resected, had only the remaining colon as a potential site for release of GLP-2. We did find that GLP-1 expression, as a proxy for GLP-2, was significantly increased in the colon of JC piglets, but not that of JI piglets. We also did observe a significant rise in plasma GLP-2 levels at the early time point, when the JC piglets were on $50 \%$ EN. Therefore, it appears that the JC piglets could produce a partial increase in endogenous GLP-2 production postresection, most likely from the colon. However, this early increase in plasma GLP-2 levels, presumably from the colon, did not appear to reach a level sufficient to promote either growth or adaptation of the intestine. A similar finding is reported in human babies, suggesting the colon does not play a significant 


\section{Articles | Hua et al.}

a

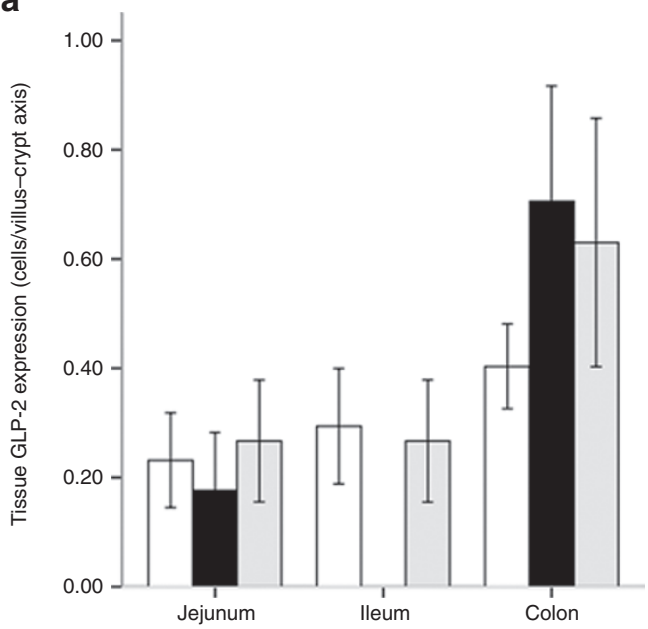

b

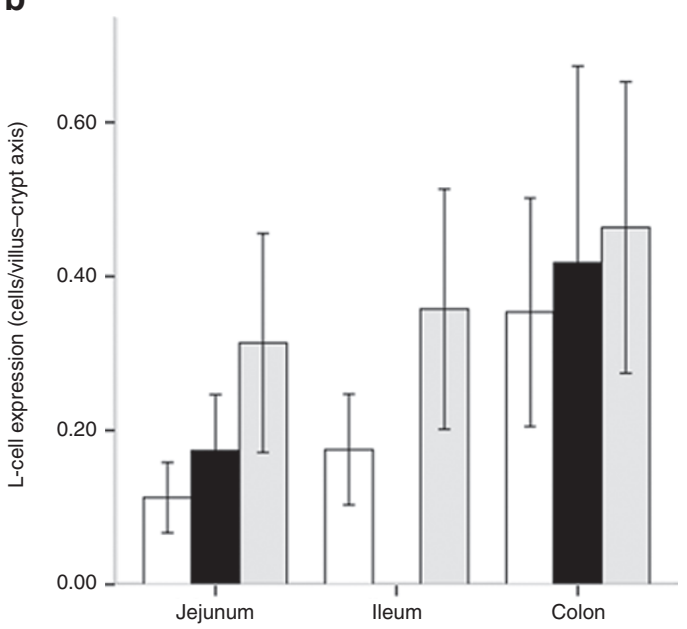

Figure 4. Tissue GLP-2 and L-cell immunohistochemical expression in the jejunum, ileum, and colon in the three surgical groups (mean \pm SE). Jl piglets, white bar, $n=16$; JC piglets, black bar, $n=17$; sham piglets, gray bar, $n=15$. (a) Tissue GLP-2 expression measured using anti-GLP-1 antibodies; (b) L-cell expression measured using anti-chromagranin A (L-cell staining) antibodies. GLP, glucagon-like peptide; JC, jejunocolic; Jl, jejunoileal.
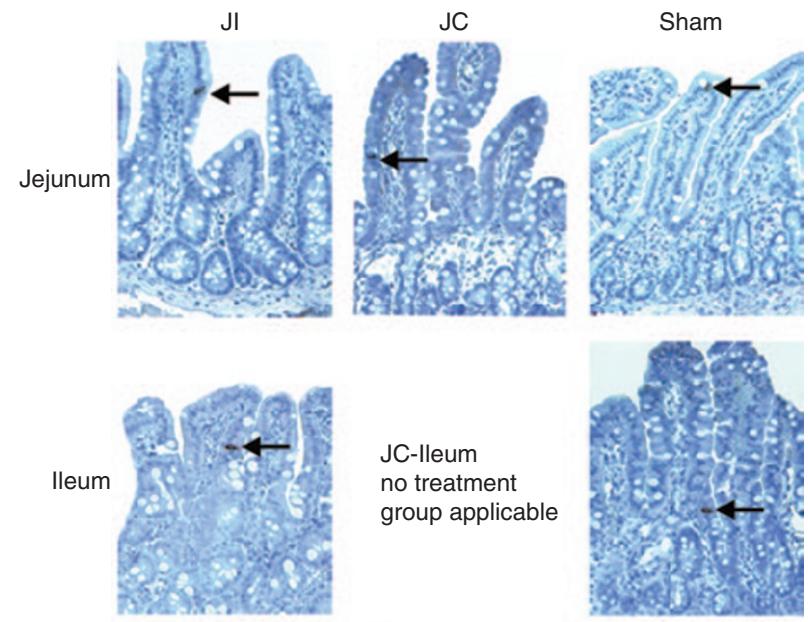

JC-Ileum no treatment group applicable
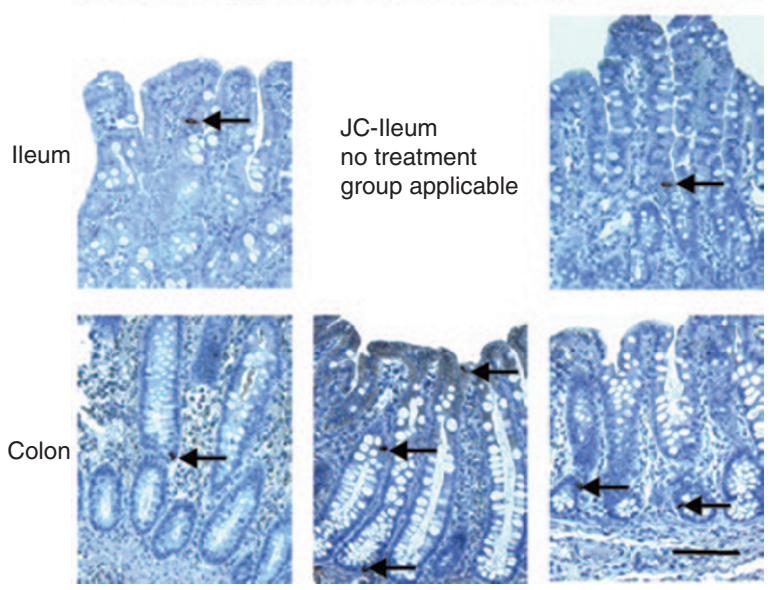

Figure 5. Representative photographs of numbers of L-cells, as detected using GLP-1 immunoreactivity in the jejunum, ileum, and colon of $\mathrm{Jl-}$, JC-, and sham-treated groups and number of GLP-1 cells per villus-crypt axis. Arrows identify diaminobenzidine (brown) stained GLP-1 cells. Bar $=100 \mu \mathrm{m}$; original magnification $\times 200$. GLP, glucagon-like peptide; JC, jejunocolic; J, jejunoileal.

role in raising plasma GLP-2 levels post-intestinal resection in neonates $(23,24)$. The developing colon may be unable to sustain progressive or adequate compensatory increase in plasma GLP-2 levels postresection, as compared with remnant ileum. This may be a key difference that contributes to the known adverse outcome from intestinal failure in babies as compared with adults. It needs to be explored further if it is at all possible to increase the residual functioning of colonic L-cells to produce GLP-2 in neonates; if this is possible, it could improve clinical outcome in this population.

Neuroendocrine L-cells are the site for GLP-2 release, yet in this study L-cell staining, while following the expected pattern in being greatest in the distal intestine, was not different among the three groups. However, plasma GLP-2 levels were different among the three groups. Therefore, it is possible that the function of L-cells is modified in the presence of intestinal resection. We do know that the expression of proglucagon in the small intestine can be regulated by nutrient exposure, in a site-specific manner, without a change in L-cell number (25). Mature rodents with $80 \%$ mid-intestinal resection have proglucagon mRNA transcription increased, without an increase in L-cells (26). However, we did not actually see an increase in GLP-1 expression as a measure of cosecreted GLP-1 and GLP-2. Because we are unable to account for actual GLP-2 secretion, it is possible that differential post-translational modification of proglucagon may be happening with the L-cells. Alternatively, differences in plasma GLP-2 levels reflect alteration in metabolism after secretion. This was not measured in this study and has not been well elucidated in animal models of intestinal resection.

Endogenous GLP-2 release is affected by many factors, in particular, the presence and amount of luminal nutrition $(14,27)$. This may be another important reason for the differences in GLP-2 release between the JI and JC piglets in our study that can be related to study design. We used a clinical model that is similar to the approach taken for infants with intestinal failure. The amount of decrease in PN calories was equal to the amount of increase in EN calories, determined by tolerance; diarrhea and weight gain were used as proxies for energy absorption from EN. In the JI piglets, the highest energy tolerated enterally was $88 \%$, whereas in JC piglets, it was only $60 \%$. This means from the time point when piglets were all fed with 50\% EN until the end of the trial, JI piglets increased the amount of luminal nutrition to an average of $\sim 90 \%$, whereas by contrast, JC piglets increased, on average, 

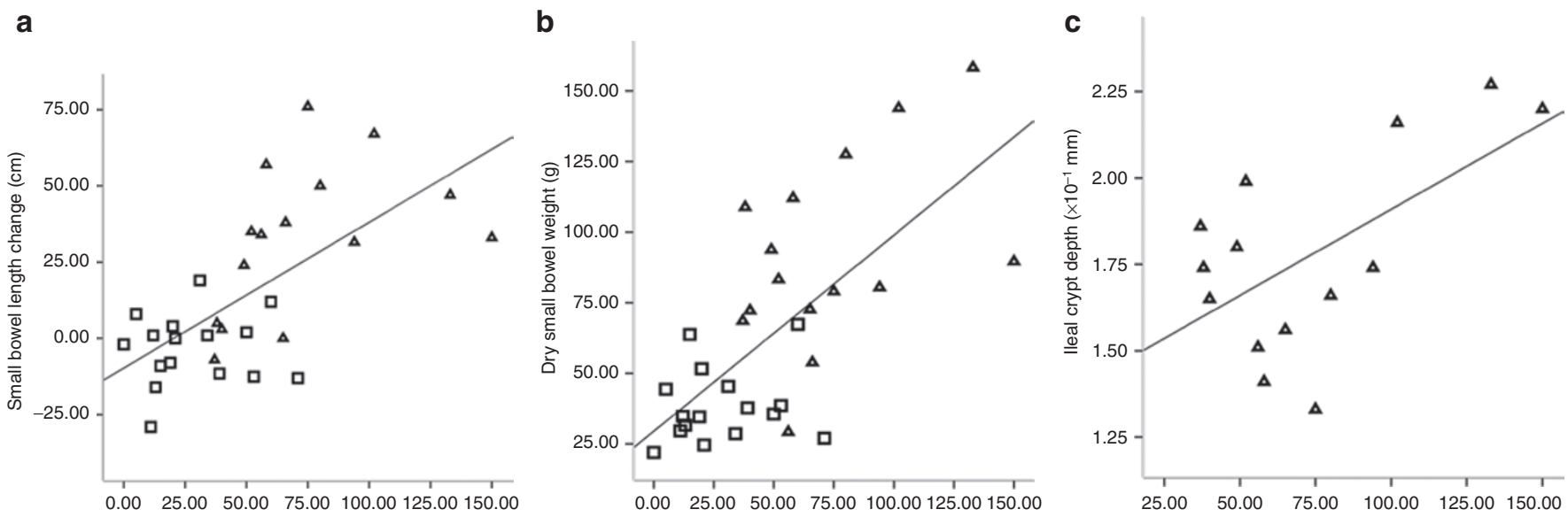

Figure 6. Correlations between GLP-2 at the end of the trial and various measures of intestinal adaptation. Jl piglets represented by triangles, $n=16$; JC piglets represented by squares, $n=17$. Correlations as follow between plasma GLP-2 and (a) small-intestinal change in length (cm), $R^{2}$ linear $=0.418 ;(\mathbf{b})$ small-intestinal dry weight $(\mathrm{g}), R^{2}$ linear $=0.454$; (c) ileum crypt depth $\left(\times 10^{-1} \mathrm{~mm}\right)$, in the Jl piglets, $R^{2}$ linear $=0.360$. Linear regression analysis, $P<0.05$. GLP, glucagon-like peptide; JC, jejunocolic; Jl, jejunoileal.

only a further $10 \%$. We recognize this as a confounder that may relate to the release of GLP-2 and to adaptation because both require luminal nutrients. Previously, Burrin et al. have shown that $60 \% \mathrm{EN}$ (percentage of total energy) was required to increase plasma GLP-2 levels in neonatal piglets fed differing proportions of EN and PN (28). This has been confirmed in other studies (29-31); therefore, the differences in EN being infused at the time GLP-2 was measured could have significantly influenced the plasma levels observed. To try to reduce this confounding effect, we did measure GLP-2 at the time all piglets were on $50 \% \mathrm{EN}$. At that time, the level in the JI piglets was still significantly higher than in the other two groups. We also measured the level at the end of trial, with EN delivered continuously at a constant rate for $24 \mathrm{~h}$, but acknowledge that the rate still varied among the three groups.

Enteral lipid is a potent nutritional stimulus of GLP-2 release (32). In addition, to differences in absolute EN, the amount of lipid being infused also was different, consistently being more for the JI piglets than for the JC piglets. Correlations between plasma GLP-2 levels and the total amount of lipid infused or the amount absorbed were also identified. In studying GLP-2 levels in human infants with intestinal resection, it was not possible to control for the amount of nutrition being delivered, and a correlation between GLP-2 levels and enteral tolerance and fat absorption was also shown (23). This is a complex issue because the role of GLP-2 in adaptation logically means enteral tolerance may be better in those piglets and infants that have higher circulating levels.

Despite this confounding issue, our data support the hypothesis that SBS piglets without ileum are deficient in GLP-2 and unable to maintain an adequate level required for adaptation and for normal growth of the intestine with development. This suggests that they may benefit from GLP-2 replacement. We propose that a trial of exogenous GLP-2 is warranted in SBS piglets, before planning human infant trials, to further elucidate the role of remnant anatomy in response to GLP2. Trials of GLP-2 treatment in short-bowel adults have had modest results to date, primarily showing improvement in stool water absorption (33). However, neonates and infants with SBS require greater nutritional support from PN, during this period of rapid growth, and therefore further translational research is needed.

In summary, in a neonatal piglet model of SBS, piglets with ileum have small-intestinal adaptation associated with progressive increase in plasma GLP-2 levels. By contrast, SBS piglets without ileum do not demonstrate intestinal adaptation or growth in length of the intestine and do not have sustained increases in plasma GLP-2 levels. The absence of ileum is a common finding in human infants with SBS who fail to undergo adaptation. These babies either succumb to complications or require transplantation. It is logical to consider, based on this translational research, that human neonates with ileal resection are GLP-2 deficient. Exogenous GLP-2 therapy should be evaluated and developed for human infants with this anatomical subtype.

\section{METHODS}

\section{Animals and Surgery}

The Faculty of Agricultural, Life and Environmental Sciences Animal Policy and Welfare Committee, University of Alberta, approved all the procedures. Male newborn Landrace-Large White cross piglets were obtained from the Swine Research and Technology Centre, University of Alberta, a bio-secure facility where the research was conducted.

All piglets were aged $5.0 \pm 1.0 \mathrm{~d}$ at the time they underwent general anesthesia for insertion of a jugular venous catheter and laparotomy for insertion of a gastrostomy feeding tube and intestinal measurement, as previously described (19). Piglets were randomly allocated into three groups: proximal intestinal resection group $(n=16)$, distal intestinal resection group $(n=17)$, and sham group $(n=15)$. The proximal resection group had a $75 \%$ mid-intestinal resection with a jejunoileal (JI) anastomosis, leaving the same amount of jejunum and ileum. The distal resection group had $75 \%$ of distal small bowel resected, including all ileum, cecum, and $5 \mathrm{~cm}$ of the colon, with a JC anastomosis. The sham group did not have intestinal resection, but otherwise had identical operative procedures. Postoperatively, piglets were housed in individual metabolic cages, in a temperaturecontrolled room, with a 12-h light/dark cycle. To prevent sepsis and gastrointestinal hemorrhage, cycled broad-spectrum antibiotics and 
acid blockade were given per protocol (19). Piglets were maintained for $14 \mathrm{~d}$ before terminal laparotomy.

\section{Nutrition}

Continuous PN infusion for all groups commenced immediately postoperatively using a sterile amino acid-based solution formulated for piglets and prepared in our laboratory (34). Prior to infusion, multivitamins (Multi-12/K 1 Pediatric, Sabex, Boucherville, Quebec, Canada), Vitamin $B_{12}$ (Abbott, Mississauga, Ontario, Canada), trace minerals, iron dextran (Ferroforte; Bimeda-MTC, Cambridge, Ontario, Canada), and lipid (Intralipid 20\%, Fresenius Kabi, Bad Homburg, Germany) were added to each PN solution bag (to create a three in one solution).

Target nutrient intake for all piglets (JI, JC, and sham) was reached on day 1 postsurgery, providing fluid $13.5 \mathrm{ml} / \mathrm{kg} / \mathrm{h}$, energy $1.1 \mathrm{MJ} /$ $\mathrm{kg} / \mathrm{d}$, amino acids $16 \mathrm{~g} / \mathrm{kg} / \mathrm{d}$, and lipid $10 \mathrm{~g} / \mathrm{kg} / \mathrm{d}$. On day 2 postoperatively, EN was started in all piglets at $10 \%$ of the total rate and was delivered continuously via the gastrostomy tube. Every $2-24 \mathrm{~h}$ the EN increased by $10 \%$ and the PN decreased the same amount. The PN and EN was isocaloric and isonitrogenous, and total nutrient delivery remained constant. A standardized feeding protocol determined advancement of EN, depending on the presence of diarrhea and daily weight gain. Only piglets without any diarrhea had the EN rate increased twice daily. EN was not increased unless intended weight gain was reached ( $\geq 50 \mathrm{~g} / \mathrm{d}$ during the first week, $\geq 100 \mathrm{~g} / \mathrm{d}$ during the second week).

\section{Fecal Fat Absorption}

Forty-eight-hour fecal collections were performed starting on days 5 and 12. Fecal effluent was collected into fitted stoma appliances (Hollister, Aurora, Ontario, Canada). EN bags were weighed for each collection period, hence, the exact enteral lipid infused was known. Fat extraction was undertaken by petroleum ether distillation using a Goldfish apparatus (method Aa 4-38, AOAC 2000) (35). Fat absorption was calculated by subtracting the fecal fat extracted from the total lipid infused, adjusted for the duration of fecal collection, and expressed as grams per kilogram per day.

\section{Radioimmunoassay}

Samples were taken for plasma GLP-2 measurement at baseline, when the EN infusion rate was increased to $50 \%$, and at the end of the trial. Measurement of plasma GLP-2 concentration in swine plasma was performed as previously described (36). The experimental detection limit was $5 \mathrm{pmol} / \mathrm{l}$, and the intra-assay coefficient of variation was $2.3 \%$ at $40 \mathrm{pmol} / \mathrm{l}$.

\section{Histology and Immunohistochemistry}

On day 14, piglets underwent a second laparotomy to measure the small-bowel length, before humane euthanasia. The entire small intestine was then removed, emptied of fecal matter, and a dry weight obtained. In each segment of small intestine, cross-sectional samples were collected for histology and immunohistochemistry analysis: $10 \mathrm{~cm}$ distal to the ligament of Treitz and $10 \mathrm{~cm}$ anterior to the ileocecal valve or anastomosis (JC). Mid-section samples of each segment of intestine were also collected in RNAlater Solution (Ambion, Burlington, Ontario, Canada): jejunum, colon, and ileum (as applicable). Paraffin blocks for $5 \mu \mathrm{m}$ intestinal sections were prepared and stained with hematoxylin and eosin using standard techniques. A micrometer eyepiece (Nikon Eclipse 80i; Nikon, Tokyo, Japan) was used by a certified animal histopathologist (P.N.N.) to measure villus height and crypt depth. Only villi observed in longitudinal sections were chosen to measure the height. The crypt depth measurement was taken in the same area as the chosen villus as often as possible. For each section, the first 10 measurements were used to calculate a mean and SD for both villus height and crypt depth.

Deparaffinized sections were used for immunohistochemistry analysis. To measure crypt cell proliferation, a monoclonal mouse anti-Ki67 primary antibody (Dako, Burlington, Canada) was used. An ultra streptavidin detection system was used to view the staining.

To measure apoptosis in the crypts and villi, an antiactive caspase- 3 antibody was used (Cell Signaling Technology, Danvers, MA). Slides were incubated with a diluted horseradish peroxidase-conjugated antirabbit IgG and counterstained with hematoxylin before viewing the staining. Only villi and crypts in longitudinal sections were examined, and a total of 10 complete villi-crypt columns were counted.

To assess the number of cells expressing GLP-2, anti-GLP-1 antibodies were used because anti-GLP-2 antibodies cross-react with many other proteins. Levels of GLP-1, concomitantly liberated from the L-cells, exactly parallel GLP-2 levels (37). Deparaffinized sections were incubated with anti-GLP-1 mouse monoclonal (1:50, Santa Cruz Biotechnology, Santa Cruz, CA) and secondary antibody (biotinylated antimouse $\operatorname{IgG}, 1: 100$, Jackson Immunoresearch, West Grove, PA). L-cells were measured after similar preparation, using primary antibody (antichromogranin A, mouse monoclonal, 1:50, Biogenex, Freemont, CA) and secondary antibody (biotinylated antimouse IgG, 1:100, Jackson Immunoresearch). Sections for GLP-1 and L-cells were visualized for reaction with diaminobenzidine (Vector Laboratories, Burlingame, CA) after counterstaining with hematoxylin. Positively stained cells per villus-crypt axis (jejunum and ileum) or per crypt (colon) were counted.

\section{GLP-2R Expression}

GLP-2R was measured using reverse-transcription PCR as previously described (38). In short, total mRNA was extracted and reverse-transcribed using Superscript-II reverse transcriptase (Invitrogen, Carlsbad, CA), and quantitative PCR was performed using Express SYBR Greener master mix with ROX (Invitrogen, Carlsbad,CA) in an ABI7900 real-time PCR thermocycler detection system (Applied Biosystems, Foster City, CA). Primer sequences (sense, 5' -ACCTTGCAGCTGATGTACAC-3'; antisense, $5^{\prime}$-GTGTTCTCCAGGTGTGCACG-3') were obtained from the University of Calgary DNA Core (Calgary, Alberta, Canada). In each intestinal segment, $18 \mathrm{~S}$ ribosomal RNA was set as the control; data are expressed as relative fold change, using the $\Delta \Delta \mathrm{Ct}$ method, to sham values.

\section{Statistics}

All data were analyzed using SPSS (version 19, IBM, New York, NY), and results are expressed as mean and SEM. Comparisons among all groups were analyzed by one-way ANOVA. Comparisons between two surgical groups were performed using independent samples Student's $t$-test. Within groups, paired-samples $t$-test was used to compare values collected at different time points. Plasma GLP-2 levels were correlated with intestinal adaptation using Pearson's correlation coefficient. An a value of $P<0.05$ was considered significant.

\section{ACKNOWLEDGMENTS}

The authors acknowledge the assistance of Rhian Ormond, Christine Pendlebury, Charlane Gorsak, and all the staff of the Swine Research and Technology Center.

\section{STATEMENT OF FINANCIAL SUPPORT}

This work was supported by grants from the SickKids Foundation; the Institute of Human Development, Child and Youth Health, Canadian Institutes of Health Research; National Grants Program (XG08-030R); Molly Towell Perinatal Research Foundation; and the Women's and Children's Health Research Institute of Alberta.

Disclosure: The authors declared no conflict of interest.

\section{REFERENCES}

1. Wales PW, de Silva N, Kim J, Lecce L, To T, Moore A. Neonatal short bowel syndrome: population-based estimates of incidence and mortality rates. J Pediatr Surg 2004;39:690-5.

2. Chaet MS, Farrell MK, Ziegler MM, Warner BW. Intensive nutritional support and remedial surgical intervention for extreme short bowel syndrome. J Pediatr Gastroenterol Nutr 1994;19:295-8.

3. Grosfeld JL, Rescorla FJ, West KW. Short bowel syndrome in infancy and childhood. Analysis of survival in 60 patients. Am J Surg 1986;151:41-6.

4. Cole CR, Hansen NI, Higgins RD, Ziegler TR, Stoll BJ; Eunice Kennedy Shriver NICHD Neonatal Research Network. Very low birth weight preterm infants with surgical short bowel syndrome: incidence, morbidity 
and mortality, and growth outcomes at 18 to 22 months. Pediatrics 2008;122:e573-82.

5. Dowling RH, Booth CC. Structural and functional changes following small intestinal resection in the rat. Clin Sci 1967;32:139-49.

6. Cisler JJ, Buchman AL. Intestinal adaptation in short bowel syndrome. J Investig Med 2005;53:402-13.

7. Ørskov C, Hartmann B, Poulsen SS, Thulesen J, Hare KJ, Holst JJ. GLP-2 stimulates colonic growth via KGF, released by subepithelial myofibroblasts with GLP-2 receptors. Regul Pept 2005;124:105-12.

8. Sigalet DL, Bawazir O, Martin GR, et al. Glucagon-like peptide-2 induces a specific pattern of adaptation in remnant jejunum. Dig Dis Sci 2006;51:1557-66.

9. Martin GR, Wallace LE, Hartmann B, et al. Nutrient-stimulated GLP-2 release and crypt cell proliferation in experimental short bowel syndrome. Am J Physiol Gastrointest Liver Physiol 2005;288:G431-8.

10. Drucker DJ. Biological actions and therapeutic potential of the glucagonlike peptides. Gastroenterology 2002;122:531-44.

11. Scott RB, Kirk D, MacNaughton WK, Meddings JB. GLP-2 augments the adaptive response to massive intestinal resection in rat. Am J Physiol 1998;275(5 Pt 1):G911-21.

12. Brubaker PL, Izzo A, Hill M, Drucker DJ. Intestinal function in mice with small bowel growth induced by glucagon-like peptide-2. Am J Physiol 1997;272(6 Pt 1):E1050-8.

13. Drucker DJ, Erlich P, Asa SL, Brubaker PL. Induction of intestinal epithelial proliferation by glucagon-like peptide 2. Proc Natl Acad Sci USA 1996;93:7911-6.

14. Roberge JN, Brubaker PL. Secretion of proglucagon-derived peptides in response to intestinal luminal nutrients. Endocrinology 1991;128:3169-74.

15. Bozkurt A, Näslund E, Holst JJ, Hellström PM. GLP-1 and GLP-2 act in concert to inhibit fasted, but not fed, small bowel motility in the rat. Regul Pept 2002;107:129-35.

16. Ljungmann K, Hartmann B, Kissmeyer-Nielsen P, Flyvbjerg A, Holst JJ, Laurberg S. Time-dependent intestinal adaptation and GLP-2 alterations after small bowel resection in rats. Am J Physiol Gastrointest Liver Physiol 2001;281:G779-85.

17. Kaji T, Tanaka H, Redstone H, Wallace LE, Holst JJ, Sigalet DL. Temporal changes in the intestinal growth promoting effects of glucagon-like peptide 2 following intestinal resection. J Surg Res 2009;152:271-80.

18. Sibbons PD, Spitz L, van Velzen D. Collateral blood flow in the distal ileum of neonatal piglets: a clue to the pathogenesis of necrotizing enterocolitis. Pediatr Pathol 1992;12:15-27.

19. Turner JM, Wales PW, Nation PN, et al. Novel neonatal piglet models of surgical short bowel syndrome with intestinal failure. J Pediatr Gastroenterol Nutr 2011;52:9-16.

20. Book SA, Bustad LK. The fetal and neonatal pig in biomedical research. J Anim Sci 1974;38:997-1002.

21. Moughan PJ, Rowan AM. The pig as a model animal for human-nutrition research. Proc Nutr Soc NZ 1989;14:116-23.

22. Larsson LI, Holst J, Håkanson R, Sundler F. Distribution and properties of glucagon immunoreactivity in the digestive tract of various mammals: an immunohistochemical and immunochemical study. Histochemistry 1975;44:281-90.

23. Sigalet DL, Martin G, Meddings J, Hartman B, Holst JJ. GLP-2 levels in infants with intestinal dysfunction. Pediatr Res 2004;56:371-6.

24. Diamond IR, Struijs MC, de Silva NT, Wales PW. Does the colon play a role in intestinal adaptation in infants with short bowel syndrome? A multiple variable analysis. J Pediatr Surg 2010;45:975-9.

25. Nian M, Gu J, Irwin DM, Drucker DJ. Human glucagon gene promoter sequences regulating tissue-specific versus nutrient-regulated gene expression. Am J Physiol Regul Integr Comp Physiol 2002;282:R173-83.

26. Fuller PJ, Beveridge DJ, Taylor RG. Ileal proglucagon gene expression in the rat: characterization in intestinal adaptation using in situ hybridization. Gastroenterology 1993;104:459-66.

27. Jeppesen PB, Hartmann B, Hansen BS, Thulesen J, Holst JJ, Mortensen PB. Impaired meal stimulated glucagon-like peptide 2 response in ileal resected short bowel patients with intestinal failure. Gut 1999;45:559-63.

28. Burrin DG, Stoll B, Jiang R, et al. Minimal enteral nutrient requirements for intestinal growth in neonatal piglets: how much is enough? Am J Clin Nutr 2000;71:1603-10.

29. Xiao Q, Boushey RP, Drucker DJ, Brubaker PL. Secretion of the intestinotropic hormone glucagon-like peptide 2 is differentially regulated by nutrients in humans. Gastroenterology 1999;117:99-105.

30. Burrin D, Guan X, Stoll B, Petersen YM, Sangild PT. Glucagon-like peptide 2: a key link between nutrition and intestinal adaptation in neonates? J Nutr 2003;133:3712-6.

31. Nelson DW, Murali SG, Liu X, Koopmann MC, Holst JJ, Ney DM. Insulinlike growth factor I and glucagon-like peptide-2 responses to fasting followed by controlled or ad libitum refeeding in rats. Am J Physiol Regul Integr Comp Physiol 2008;294:R1175-84.

32. Holst JJ, Christiansen J, Kühl C. The enteroglucagon response to intrajejunal infusion of glucose, triglycerides, and sodium chloride, and its relation to jejunal inhibition of gastric acid secretion in man. Scand J Gastroenterol 1976;11:297-304.

33. Jeppesen PB, Hartmann B, Thulesen J, et al. Glucagon-like peptide 2 improves nutrient absorption and nutritional status in short-bowel patients with no colon. Gastroenterology 2001;120:806-15.

34. Wykes LJ, Ball RO, Pencharz PB. Development and validation of a total parenteral nutrition model in the neonatal piglet. J Nutr 1993;123:1248-59.

35. AOAC International. Official Methods of Analysis of AOAC International. Arlington, VA: Association of Analytical Chemists, 2000.

36. Hartmann B, Johnsen AH, Orskov C, Adelhorst K, Thim L, Holst JJ. Structure, measurement, and secretion of human glucagon-like peptide-2. Peptides 2000;21:73-80.

37. Orskov C, Holst JJ, Knuhtsen S, Baldissera FG, Poulsen SS, Nielsen OV. Glucagon-like peptides GLP-1 and GLP-2, predicted products of the glucagon gene, are secreted separately from pig small intestine but not pancreas. Endocrinology 1986;119:1467-75.

38. Petersen YM, Burrin DG, Sangild PT. GLP-2 has differential effects on small intestine growth and function in fetal and neonatal pigs. Am J Physiol Regul Integr Comp Physiol 2001;281:R1986-93. 